Recepción: 01 / 03/ 2018

Aceptación: 15 / 05 / 2018

Publicación: 07 / 09 / 2018
Ciencias de la salud

Artículo Científico

\title{
Tratamiento farmacológico en pacientes con problemas cardiovascular
}

\author{
Pharmacological treatment in patients with cardiovascular problems \\ Tratamiento farmacológico en pacientes con problemas cardiovascular
}

\author{
Cristhian R. Vallejo-Zambrano ${ }^{\mathrm{I}}$ \\ cristhian_564@hotmail.com \\ Jordi A.Chonillo-Franco II \\ Jordichonillo58@yahoo.com \\ Linda S. Vera-Garces III \\ linda_12ju@hotmail.com
}

\author{
Leonor D. Loor-Loor ${ }^{\mathrm{IV}}$ \\ leonordloor_12r@yahoo.com \\ Gustavo A. Munizaga-Marquez ${ }^{\mathrm{V}}$ \\ Gustavomarquez2@gmail.com \\ Rosa G. Medranda-Zambrano VI \\ rossabella@gmail.com
}

Correspondencia: cristhian_564@hotmail.com

\footnotetext{
I. Médico Cirujano, Centro de Salud tipo B, Guayaquil, Ecuador.

II. Médico Cirujano. Hospital Básico, Misereor, Gualaquiza, Ecuador.

III. Médico Cirujano, Centro de Salud el Limón, el Empalme, Guayas, Ecuador.

IV. Médico Cirujano, Centro de Salud tipo B, Manta, Ecuador.

v. Médico Cirujano, Centro de Salud tipo B, Manta, Ecuador.

VI. Médico Cirujano, Hospital General Rodríguez Zambrano, Manta, Ecuador.
} 
Cristhian R. Vallejo-Zambrano; Jordi A.Chonillo-Franco; Linda S. Vera-Garces; Leonor D. Loor-Loor; Gustavo A. Munizaga-Marquez; Rosa G. Medranda-Zambrano

\section{Resumen}

El presente artículo se explora la importancia de los factores que influyen en la adherencia o no adherencia a tratamientos farmacológicos y no farmacológicos en pacientes con problemas de enfermedad cardiovascular, los factores que influyen en la adherencia son condicionantes que afectan el comportamiento de la persona hacia el seguimiento de las recomendaciones de medicación dadas por el personal de salud y entre esta están las características socioeconómicas, las relacionadas con la asistencia dada por el sistema sanitario, las relacionadas con la enfermedad, con el tratamiento terapéutico y con el paciente. En cuanto a la adherencia a los tratamientos farmacológicos corresponde al grado de comportamiento de una persona para tomar los medicamentos, si corresponde con las recomendaciones dadas por el asistente sanitario. Con respecto a tratamientos no farmacológicos responde al grado de coincidencia entre el comportamiento del paciente y las recomendaciones dadas por el profesional de la salud con respecto al cambio de hábitos de vida del paciente (dejar el sedentarismo, de beber o de fumar, etc.). Los pacientes con factores de riesgo cardiovascular son personas que presentan condiciones en forma temprana que se asocian con la probabilidad de desarrollar enfermedad coronaria o cardiovascular en un determinado periodo.

Palabras claves: Enfermedad cardiovascular, adherencia, tratamiento farmacológico, hábitos de vida del paciente, factores de riesgo. 


\section{Abstract}

This article explores the importance of the factors that influence the adherence or non-adherence to pharmacological and non-pharmacological treatments in patients with cardiovascular disease problems. The factors that influence adherence are conditioning factors that affect the behavior of the person towards the follow-up of the medication recommendations given by the health personnel and among these are the socioeconomic characteristics, those related to the assistance given by the health system, those related to the disease, with the therapeutic treatment and with the patient. Regarding the adherence to pharmacological treatments corresponds to the degree of behavior of a person to take medications, if appropriate with the recommendations given by the health assistant. Regarding non-pharmacological treatments, it responds to the degree of coincidence between the patient's behavior and the recommendations given by the health professional regarding the change in the patient's life habits (leaving sedentary lifestyle, drinking or smoking, etc.). Patients with cardiovascular risk factors are people who have conditions in an early form that are associated with the probability of developing coronary or cardiovascular disease in a certain period.

Keys words: Cardiovascular disease, adherence, pharmacological treatment, patient's life habits, risk factors. 
Cristhian R. Vallejo-Zambrano; Jordi A.Chonillo-Franco; Linda S. Vera-Garces; Leonor D. Loor-Loor; Gustavo A. Munizaga-Marquez; Rosa G. Medranda-Zambrano

\section{Introducción.}

A través de la historia se ha indicado que una de las mejores defensas para luchar contra la enfermedad es el empleo de medicamentos, a través del tratamiento farmacológico. Donde la estrategia es lo mejor para optimizar las condiciones de los pacientes con enfermedades que se asocian con problemas cardiovasculares, destacan la importancia de los tratamientos farmacológicos prescritos. Algunas son de carácter personal del usuario, como podría ser la disposición para el autocuidado, y otras relacionadas con las situaciones del régimen saludable.

Las enfermedades cardiovasculares constituyen un problema de salud de primer orden. En todo el mundo 16.7 millones de muertes se deben a dichas enfermedades. Además, cinco de las diez principales amenazas mundiales para la salud se relacionan con las enfermedades no transmisibles, como la hipertensión arterial, el tabaquismo, el consumo de alcohol, la hipercolesterolemia y la obesidad o el sobrepeso. (OMS, 2013)

El aumento de enfermedades crónicas, tales como enfermedades cardiovasculares (enfermedad isquémica del corazón, enfermedad cerebro vascular, hipertensión), cáncer, enfermedades pulmonares crónicas, entre otras, está determinado por los estilos de vida. (Mejía, Serna, Sierra, \& Muñoz, 1999)

Su íntima relación causal con el conjunto de las enfermedades cardiovasculares (ECV), la hipertensión arterial (HTA) es el primer factor de riesgo para la mortalidad y el tercero más importante como contribuyente a la discapacidad en todo el mundo. (Ortiz, Vaamonde, \& Zorrilla, 2011) (Díez Tejedor, Fuentes, Gil Núñez, \& Gil Peralta, 2006) 
La Organización Mundial de la Salud (OMS) estima que, en el caso de la hipertensión, el porcentaje de pacientes que cumplen con el régimen terapéutico es solo del $27 \%$ en Gambia, $46 \%$ en China, y $51 \%$ en los Estados Unidos. Se calcula que el porcentaje de pacientes tratados que tienen un buen control de la tensión arterial es sólo del 7\% en el Reino Unido y 4,5\% en Venezuela. En Sudán, solo el $18 \%$ de los pacientes que no tienen una buena adherencia al tratamiento logran un buen control de la tensión arterial; mientras que, aquellos que tienen una buena adherencia, el porcentaje de pacientes controlados alcanza el 96\%. Varios análisis rigurosos, que se recogen en un estudio de la OMS, revelan que la observancia del tratamiento por parte de los pacientes con enfermedades crónicas, en los países desarrollados, es del 50\%. Los datos disponibles señalan que la adherencia es mucho menor en los países en desarrollo. (Renato Zambrano, Duitama M, \& Posada, 2012)

Una vez establecida la enfermedad cardiovascular, puede acompañar a la persona durante mucho tiempo, empeorar su estado o conducirlo a la muerte; sin embargo, el comportamiento de las personas podrá desempeñar un papel importante en esta evolución e incluso alargar su vida, si se adhiere adecuadamente al estilo de vida que exige la enfermedad cardiovascular. (Vlasnik, Aliotta, \& DeLor, 2005)

Gran parte de esta problemática se encuentra relacionada con la falta de adherencia a los tratamientos farmacológicos y no farmacológicos, reflejada en un gran número de consecuencias no solamente en la salud, sino familiares y económicas. Por ende, la iniciativa mundial lanzada en 2001 por el grupo de Enfermedades No Transmisibles y Salud Mental de la OMS, en el trabajo realizado bajo el marco de un proyecto sobre adherencia terapéutica a largo plazo, generó un informe técnico divulgado entre 2003 y 2004, OMS. (Rodríguez Acelas \& Gómez Ochoa, 2010) 
Cristhian R. Vallejo-Zambrano; Jordi A.Chonillo-Franco; Linda S. Vera-Garces; Leonor D. Loor-Loor; Gustavo A. Munizaga-Marquez; Rosa G. Medranda-Zambrano

La definición de cumplimiento o adherencia terapéutica se da por la conducta del paciente en relación con la toma de medicación, el seguimiento de una dieta o la modificación de su estilo de vida, que coinciden con las indicaciones dadas por su médico. (Haynes, Sackett, Taylor, \& Show, 1979)

Según (Kontz, 1991) "la adherencia es un fenómeno complejo en el que un individuo muestra comportamientos consistentes con su propia definición de salud y con las actividades relacionadas con la salud".

De acuerdo con la OMS, la adherencia "es el grado en que el comportamiento de una persona -tomar el medicamento, seguir un régimen alimentario y ejecutar cambios del modo de vida- se corresponde con las recomendaciones acordadas de un prestador de asistencia sanitaria". (Sabaté, 2003)

El incremento de la eficacia de las acciones destinadas a que la persona cumpla con el tratamiento puede tener un mayor impacto en su salud que cualquier mejora en un tratamiento médico específico. (Cramer \& Mattson, 1991)

El nivel de control y tratamiento continúa siendo mejorable. Esta situación está determinada por una falta de detección de la enfermedad, fallos en la adecuación del tratamiento establecido y la baja adherencia al tratamiento. (Messerli, Williams, \& Ritz , 2007)

(Orueta, Toledanob, \& Gómez-Calcerradaa, 2008) hacen referencia al papel preponderante que ejerce la motivación en el proceso de la adherencia y la fuerte influencia de factores como las creencias, los valores, la sintomatología percibida, la relación médico-paciente, el apoyo familiarsocial, entre otros. 
(Sabaté, 2003), la adherencia es un fenómeno multidimensional determinado por la acción recíproca de cinco factores, con los cuales los pacientes interactúan: factores socioeconómicos, factores relacionados con los sistemas de salud, factores relacionados con la enfermedad, factores relacionados con la terapia y factores relacionados con el paciente.

(Bonilla Ibáñez, 2007) diseño un "Instrumento para evaluar los factores que influyen en la adherencia a tratamientos farmacológicos y no farmacológicos en pacientes con factores de riesgo de enfermedad cardiovascular".

En relación a lo expuesto se hace imprescindible que la enfermería valore en los pacientes con problemas cardiovasculares los factores que están relacionados con el tratamiento farmacológico.

\section{Metodología.}

El presente estudio científico tiene una visión directamente específica, se hizo la revisión de literatura más próxima sobre el tratamiento farmacológico en pacientes con problemas cardiovasculares. Para la realización de la actual investigación, se considero, información confiable sobre trabajos figurados, versados en este tema, se utilizaron informaciones bibliográficas, se la obtuvo de libros electrónicos, consultas electrónicas, artículos científicos y revistas.

\section{Desarrollo.}

Factores que influyen en la adherencia al tratamiento farmacológico y no farmacológico

Características que afectan el comportamiento de la persona hacia el seguimiento de la medicación o recomendaciones dadas por el personal de salud. Entre ellas están las características 
Cristhian R. Vallejo-Zambrano; Jordi A.Chonillo-Franco; Linda S. Vera-Garces; Leonor D. Loor-Loor; Gustavo A. Munizaga-Marquez; Rosa G. Medranda-Zambrano

socioeconómicas, las relaciones con la enfermedad, con el tratamiento, con el paciente y el sistema de atención sanitaria.

Conjunto de factores internos y externos que el paciente en situación de riesgo de enfermedad cardiovascular percibe como influyente en cierto grado, respecto al cumplimiento de los tratamientos y sugerencias dadas por profesionales de la salud.

Adherencia a los tratamientos farmacológicos

Estado acerca del comportamiento de una persona respecto de cómo tomar los medicamentos; se corresponde con las recomendaciones acordadas con un prestador de asistencia sanitaria. (Rodríguez Acelas \& Gómez Ochoa, 2010)

Brannon sostiene que uno de los predictores con mayor peso en la adherencia al tratamiento es el grado de apoyo social que se tiene por parte de los amigos y la familia; en general, las personas que se sienten aisladas de los demás tienden a no ser cumplidoras, mientras que aquellas que disfrutan de relaciones personales cercanas están más dispuestas a seguir con el tratamiento prescrito. (Brannon, 2002)

Reportó que la adherencia al tratamiento en un alto porcentaje de pacientes hipertensos se asocio al apoyo proporcionado por la familia. Por lo tanto, el apoyo social se constituye en un factor fundamental en el logro de las metas terapéuticas. (Contreras Orozco, 2010)

De acuerdo con el documento publicado por la OMS, "Adherencia a los tratamientos a largo plazo: Pruebas para la acción”, debido a la deficiente adherencia al tratamiento antihipertensivo, aproximadamente $75 \%$ de los pacientes con diagnóstico de hipertensión arterial en Estados Unidos no logran el control óptimo de la presión arterial. (Burt, 1995) (Costa, 1996) 
Grado en que el comportamiento de una persona, como tomar los medicamentos, se corresponde con las recomendaciones acordadas con un prestador de asistencia sanitaria. (FLÓREZ TORRES, 2009)

Grado al cual el comportamiento de un paciente con factores de riesgo de enfermedad cardiovascular corresponde a las recomendaciones hechas por un proveedor de cuidado de salud, sea médico o personal de enfermería. Estas recomendaciones se relacionan con medicamentos: tipo, dosis, frecuencia, duración del tratamiento y recomendaciones sobre su administración en relación con horario de comidas, horarios diurnos y otros. (Bonilla Ibáñez, 2007)

Los resultados reflejan un grupo comprometido con el cuidado de su salud y un sistema de atención que promueve en los pacientes comportamientos positivos que favorecen la adherencia terapéutica. Sin embargo, se hace necesario fortalecer en el $52 \%$ de los participantes, las estrategias de afrontamiento de la enfermedad que permita un mejor control de los estados de angustia identificados. (Contreras Orozco, 2010)

Estos hallazgos permiten destacar la importancia de la relación paciente-proveedor en el grado de adherencia de los pacientes, haciendo necesario que la institución y el equipo de salud y enfermería enfaticen las acciones de autocuidado con apoyo, acompañamiento permanente y orientación acorde a sus necesidades, a sus expectativas y a las características individuales de las personas atendidas. (Ortega Oviedo \& Vargas Rosero, 2014)

El seguimiento farmacoterapéutico (SFT) y la educación sanitaria mejoran la adherencia al tratamiento. Además ser paciente cumplidor al final del estudio se relaciona con la mejora de los objetivos de la presión arterial y de presión arterial/colesterol total. (Rodríguez Acelas \& Gómez Ochoa, 2010) 
Cristhian R. Vallejo-Zambrano; Jordi A.Chonillo-Franco; Linda S. Vera-Garces; Leonor D. Loor-Loor; Gustavo A. Munizaga-Marquez; Rosa G. Medranda-Zambrano

Se produce un mayor abandono de los tratamientos cardiovasculares originados en atención primaria, en relación con una mayor prescripción en dicho nivel de fármacos de utilidad terapéutica baja. En el abandono de la medicación intervienen tanto las variables sociodemográficas de los pacientes como las características del tratamiento. (García-Reyes, López-Torres, \& García, 2002)

\section{Adherencia a los tratamientos no farmacológicos}

Grado de coincidencia entre el comportamiento del paciente y las recomendaciones dadas por el profesional de salud en cuanto al cambio de hábitos de vida (dejar el sedentarismo, dejar de beber o de fumar, etc.). (FLÓREZ TORRES, 2009)

Estado acerca del comportamiento de una persona de cómo seguir un régimen alimentario y ejecutar cambios en los hábitos diarios de vida; se corresponde con las recomendaciones acordadas con un prestador de asistencia sanitaria, Rodríguez y Gómez, (2010).

Grado al cual el comportamiento de un paciente con factores de riesgo de enfermedad cardiovascular corresponde a las recomendaciones hecha por un proveedor de cuidado de salud, sea médico o personal de enfermería. Estas recomendaciones no farmacológicas corresponden a cambios de dieta, control de peso, aumento y regulación de ejercicio físico, supresión o disminución en el hábito de fumar o consumo de alcohol. Bonilla, (2007).

\section{Pacientes con factores de riesgo de enfermedad cardiovascular}

Personas con características o condiciones presentes en forma temprana que se asocian con la probabilidad de desarrollar enfermedad coronaria o cardiovascular en un periodo determinado. Dichas condiciones están constituidas por hipertensión arterial, diabetes, obesidad, dislipidemia, tabaquismo y sedentarismo, Rodríguez y Gómez, (2010). 
Se identifica a las personas con factores de riesgo cardiovascular aquellos que presentan condiciones de: hipertensión arterial, diabetes, sedentarismo, obesidad, hipercolesterolemia, dislipidemia, alcohol y tabaquismo, Bonilla, (2007).

El riesgo cardiovascular absoluto de los hipertensos no controlados en atención primaria en España es muy relevante. Más de la mitad de los pacientes mantienen riesgo alto o muy alto, con elevada prevalencia de LOD y ECS. La actitud terapéutica ante estos pacientes sigue siendo conservadora, aunque se observa mejoría respecto a estudios anteriores, Márquez, et al. (2007).

Estudios epidemiológicos según, Lewington, (2003), han señalado las enfermedades cardiovasculares (ECV) como la primera causa de morbimortalidad en los países desarrollados.

La hipertensión arterial (HTA) es el principal factor de riesgo para el desarrollo de enfermedades cardiovasculares (ECV), Burt, et al. (2003).

El porcentaje de incumplimiento en el tratamiento farmacológico de la HTA en España es alto, aunque de forma global se observa un ligero descenso en los últimos estudios, Márquez, et al. (2002).

El 55\% de los pacientes con hipertensión arterial tenían controlada la PA, independientemente de la presencia de ECV. Los estilos de vida y alimentación, especialmente el tabaquismo y la obesidad, se relacionan independientemente con la falta de control de la PA, Cordero, et al. (2011).

La prevalencia de hipertensión en España es alta y un importante porcentaje de pacientes hipertensos aún están sin diagnosticar. La hipertensión se asoció con diabetes y prediabetes, y aunque el tratamiento farmacológico es cada vez más frecuente, no logra mejorar el grado de 
Cristhian R. Vallejo-Zambrano; Jordi A.Chonillo-Franco; Linda S. Vera-Garces; Leonor D. Loor-Loor; Gustavo A. Munizaga-Marquez; Rosa G. Medranda-Zambrano

control, que continúa siendo bajo. Es importante desarrollar y promocionar campañas poblacionales de prevención, detección y tratamiento de la hipertensión arterial, Menéndez, et al. (2016).

Según Macías (1995), Dueñas y Noval, (1995), para lograr disminuciones importantes en la mortalidad por ECV debe controlarse a más del $85 \%$ de los hipertensos.

\section{Factores de riesgo de enfermedad cardiovascular}

Entre los factores de riesgo que afectan al comportamiento de las personas con problemas cardiovasculares están los factores socioeconómicos, factores relacionados con los sistemas de salud, factores relacionados con la enfermedad, factores relacionados con la terapia y factores relacionados con el paciente.

\section{Factores socioeconómicos}

La presencia de factores socioeconómicos que influyen negativamente en la adherencia al tratamiento, debido principalmente al bajo nivel educativo y a la poca disponibilidad económica para cubrir los gastos que requiere el tratamiento, lo cual es compatible con las características de los participantes. Por tanto, es importante tener en cuenta que el perfil de bajo nivel socioeconómico pone en evidencia el riesgo de no poder responder con los comportamientos de adherencia a los tratamientos, Herrera, (2012).

Reconocimiento en las personas de la importancia de adherirse a los tratamientos, presencia de redes de apoyo de la familia y grupos de amigos, la disponibilidad económica, apoyo social, ocupación laboral, dificultad para mantener las necesidades básicas, tiempo y permisos laborales para asistir a la institución de salud y continuidad en el tratamiento, Rodríguez y Gómez, (2010). 
La disponibilidad de recursos para la satisfacción de las necesidades básicas, la consecución oportuna de los medicamentos, el estado de preparación de la familia, las redes de apoyo son intervenciones sociales efectivas, influyeron de manera positiva en la adherencia, Flores, (2009).

Contar con el apoyo de la familia los anima a continuar con el tratamiento, lo cual favorece de manera positiva la adherencia terapéutica; existen pruebas sustanciales de que el apoyo de los compañeros de los pacientes puede mejorar la adherencia y reducir la cantidad de tiempo dedicado por los profesionales de la salud a la atención de los procesos crónicos, OMS, (2004).

\section{Factores relacionados con los sistemas de salud}

Proporcionar la información necesaria de modo que favorezca y garantice niveles mínimos de comprensión por parte del enfermo contribuye a mejorar la adherencia. De igual forma, la satisfacción del paciente, desde el punto de vista afectivo, con la relación establecida con el terapeuta se ha asociado a un notable incremento del cumplimiento terapéutico, Arias, (2001).

Los procedimientos propios del sistema de salud, la disponibilidad y accesibilidad de los servicios, el apoyo a la educación de los pacientes, no obstante se reportaron fallas relacionadas con la comunicación e información escrita sobre las recomendaciones dadas, Flores, (2009).

Aprobación hacia la institución y los servicios que presta, por parte del cliente interno y externo, necesidad de fortalecer la educación en los pacientes, visualizándose falencias en conocimientos de los participantes en cuanto a la enfermedad, tratamientos, controles y cuidados, Rodríguez y Gómez, (2010). 
Cristhian R. Vallejo-Zambrano; Jordi A.Chonillo-Franco; Linda S. Vera-Garces; Leonor D. Loor-Loor; Gustavo A. Munizaga-Marquez; Rosa G. Medranda-Zambrano

Factores relacionados con la enfermedad

Las condiciones de salud de los pacientes influyeron de manera positiva en la adherencia, que fue favorecida por la importancia dada al tratamiento para manejar su salud, el conocimiento acerca de la enfermedad y la motivación para tratarla. A pesar de esto, la percepción de la gravedad de la enfermedad afecta de manera negativa los comportamientos de adherencia, Flores, (2009).

Para la Organización Mundial de la Salud, con relación a las características de la enfermedad es necesario señalar la importancia de los síntomas como clave para la acción y como reforzadores de la adherencia. El paciente que experimenta un conjunto particular de síntomas perturbadores y un alivio inmediato para estos síntomas al adherirse a las prescripciones médicas y de enfermería, tiene mayores posibilidades de desarrollar un buen nivel de adherencia; mientras que el paciente que presenta una enfermedad asintomática no dispone de claves internas para la acción y su seguimiento de la prescripción no recibe refuerzo, Roter, et al. (1998)

Los pacientes con factores de riesgo de enfermedad cardiovascular, intervinieron positivamente en la adherencia, debido al conocimiento que tienen los pacientes acerca de su enfermedad y la motivación para tratarla, Herrera, (2008).

Factores relacionados con la terapia

Estudios respecto a la dosificación, duración y efectos secundarios de los tratamientos y su relación con la conducta de cumplimiento arrojaron que los regímenes de medicación con monodosis dan lugar a mejores tasas de adherencia que los regímenes multidosis, y que también disminuía a medida que la terapia se alargaba y provocaba mayor cantidad de efectos secundarios indeseados, Hernández, (1998). 
En estos paciente influyeron de manera positiva en la adherencia terapéutica; se encontró un gran porcentaje de actitudes positivas hacia el cumplimiento de los tratamientos farmacológicos y hacia las recomendaciones para mejorar la salud mediante el control del peso, la dieta y el ejercicio, Flores, (2009).

Con relación al régimen terapéutico, entre las características o aspectos que ejercen una mayor influencia tenemos la complejidad, la dosificación y los efectos secundarios de los tratamientos, Jarosz, et al. (2000).

Confianza en los beneficios del tratamiento, falta de compromiso de las personas para cambiar algunos hábitos, como la alimentación y la actividad física, Rodríguez y Gómez, (2010).

Cuanto más complejo sea el tratamiento (si exige cambios en las actividades habituales de la vida cotidiana, varios medicamentos, diversos horarios), más dificultades implicará para las respuestas de adherencia del paciente, Dailey, Kim, Lian, (2001).

\section{Factores relacionados con el paciente}

Guardan relación con el conocimiento acerca de la enfermedad y la importancia de seguir el tratamiento para mejorar su salud, influyen de manera positiva hacia la adherencia, Flores, (2009).

Importancia de continuar su tratamiento para mejorar su salud, pensamientos que en ocasiones generan sentimientos de inseguridad en su recuperación, Rodríguez y Gómez, (2010).

Según la Organización Mundial de la Salud, las percepciones que tiene el paciente sobre la necesidad personal de medicación son influenciadas por los síntomas, las expectativas y experiencias y por los conocimientos de la enfermedad. Las inquietudes por la medicación surgen de 
Cristhian R. Vallejo-Zambrano; Jordi A.Chonillo-Franco; Linda S. Vera-Garces; Leonor D. Loor-Loor; Gustavo A. Munizaga-Marquez; Rosa G. Medranda-Zambrano

manera característica de las creencias acerca de los efectos colaterales y la interrupción del modo de vida, y de preocupaciones más abstractas sobre los efectos a largo plazo y la dependencia, OMS, (2004).

\section{Conclusiones.}

Es importante que los médicos y profesionales de enfermería identifiquen las diversas dimensiones que presentan los pacientes con problemas cardiovasculares frente a la adherencia o no adherencia a los tratamientos farmacológicos y no farmacológicos, a fin de contribuir a establecer las estrategias que mejoren la adherencia en pacientes con riesgo cardiovascular. En cierta manera se sugiere transmitir las recomendaciones médicas en sus prescripciones a fin de que orienten a los pacientes de múltiples maneras una terapia adaptada a cada uno de sus requerimientos con el fin de contribuir y con un lenguaje sencillo evitando el uso intensivo de tecnicismo y reducir las complicaciones. También es fundamental la organización y la asistencia a los pacientes a fin de garantizar una educación sobre la nutrición de manera suficiente recomendando la importancia del establecimiento de procedimientos más seguros para el manejo de la dieta, con el propósito que los pacientes puedan incorporar a su estilo de vida las características principales de la terapia nutricional específica. Conviene considerar los diversos factores que interactúan en el comportamiento de los pacientes con problemas cardiovasculares, como la disponibilidad de recurso económicos para satisfacer las necesidades básicas, de redes de apoyo familiar, la disponibilidad de equipos y un sistema de salud organizado que responda a las preocupaciones y alcance de los tratamientos farmacológico y no farmacológico, el buen trato y la claridad con que la enfermera y el médico les explican todo lo relacionado con su enfermedad. 


\section{Bibliografia.}

Bonilla Ibáñez, C. (2007). Diseño de un instrumento para evaluar los factores que influyen en la adherencia a tratamientos, en personas que presentan factores de riesgo de enfermedad cardiovascular. Avances en Enfermería, 25(1), 46-55.

Brannon, L. (2002). Psicología de la salud. Madrid: Paraninfo-Thomson Learning.

Burt, V. (1995). Prevalence of hypertension in the US adult population results from the Third National Health and Nutrition Examination Survey, 1988-1991. Hypertension, 26(2), 201211.

Contreras Orozco, A. (2010). Factores que influyen en la adherencia a tratamientos farmacológicos y no farmacológicos en los pacientes inscritos en el Programa de Control de la Hipertensión Arterial de la Unidad Básica de Atención de COOMEVA. Sincelejo (Colombia), 2006. Salud Uninorte. Barranquilla (Col.), 26(2), 201-211.

Costa, F. (1996). Compilance with antihypertensive treatment. Clinical \& Experimental Hypertension, 18(1), 463-472.

Cramer, J., \& Mattson, R. (1991). Monitoring compliance with antiepileptic Drug therapy. En J. Cramer, \& B. Spilker, Patient compliance in medical practice and Clinical Trials (págs. 123- 137). Nueva York: Raven Press.

Díez Tejedor, E., Fuentes, B., Gil Núñez, A., \& Gil Peralta, A. (2006). Guía para el tratamiento preventivo de la isquemia cerebral. En E. Díez Tejedo, Guía para el tratamiento y prevención del ictus (págs. 133-183). Barcelona: Prous Science.

FLÓREZ TORRES, I. (2009). Adherencia a tratamientos en pacientes con factores de riesgo cardiovascular. Avances en Enfermería, 27(2), 25-32.

García-Reyes, M., López-Torres, J., \& García, E. (2002). Cumplimiento terapéutico en pacientes con enfermedades cardiovasculares. Medicina Clínica, 118(10), 360-400.

Haynes, R., Sackett, D., Taylor, W., \& Show, J. (1979). Annotated and indexed bibliography on compliance of the therapeutic and preventive regimens. En R. Haynes, W. Taylor, \& D. Sackett, Compliance in health care (págs. 76-81). Johns Hopkins University Press: Baltimore.

Kontz, M. (1991). Proposed model for assessing compliance within the unitary man/human framework based on an analysis of the concept of compliance. En R. Carroll-Johnson, Classification of Nursing Diagnoses, Proceedings of the Ninth Conference (págs. 161-171). Philadelphia: Lippincott.

Mejía, E., Serna, L., Sierra, T., \& Muñoz, A. (1999). Descripción de actitudes y prácticas de estilo de vida saludables en médicos y personal de enfermería, Metrosalud. Rev Epidemiol Antioquia, 24(3-4), 195-206. 
Cristhian R. Vallejo-Zambrano; Jordi A.Chonillo-Franco; Linda S. Vera-Garces; Leonor D. Loor-Loor; Gustavo A. Munizaga-Marquez; Rosa G. Medranda-Zambrano

Messerli, F., Williams, B., \& Ritz , E. (2007). Essential hypertension. Lancet, 370(9587), 591-603.

OMS. (2013). Información general sobre la HIPERTENSIÓN en el mundo. Ginebra: Organización Mundial de la Salud.

Ortega Oviedo, S., \& Vargas Rosero, E. (2014). Grado de adherencia a tratamientos en personas con riesgo cardiovascular. Avances en Enfermería, 32(1), 25-32.

Ortiz, H., Vaamonde, R., \& Zorrilla, B. (2011). Prevalencia, grado de control y tratamiento de la hipertensión arterial en la población de 30 a 74 años de la Comunidad de Madrid. Estudio PREDIMERC. Revista Española de Salud Pública, 329-338.

Orueta, R., Toledanob, R., \& Gómez-Calcerradaa, M. (2008). Cumplimiento terapéutico. SEMERGEN - Medicina de Familia, 34(5), 235-243.

Renato Zambrano, C., Duitama M, J., \& Posada, J. (2012). Percepción de la adherencia a tratamientos en pacientes con factores de riesgo cardiovascular. Revista Facultad Nacional de Salud Pública, 30(2), 163-174.

Rodríguez Acelas, A., \& Gómez Ochoa, A. (2010). Factores influyentes en adherencia al tratamiento en pacientes con riesgo cardiovascular. Avances en Enfermería, 28(1), 63-71.

Sabaté, E. (2003). Adherence to long-term therapies: Evidence for action ( Illustrated ed.). Ginebra: World Health Organization.

Vlasnik, J., Aliotta, S., \& DeLor, B. (2005). Medication adherence: factors influencing compliance with prescribed medication plans. Case Manager, 16(2), 47-51. 